\begin{tabular}{|c|c|}
\hline Title & The J6JFH1 Strain of Hepatitis C V irus Infects Human B-Cells with Low Replication Efficacy \\
\hline Author(s) & Nakai, Masato; Seya, T sukasa; Matsumoto, Misako; Shimotohno, Kunitada; Sakamoto, Naoy a; A ly, Hussein H. \\
\hline Citation & $\begin{array}{l}\text { Viral Immunology, 27(6), 285-294 } \\
\text { https://doi.org/10.1089/Nim.2013.0140 }\end{array}$ \\
\hline Issue Date & 201408 \\
\hline Doc URL & http:/hdl.handle.net/2115/56871 \\
\hline Rights & $\begin{array}{l}\text { This is a copy of an article published in the V iral Immunology @ } 2014 \text { copyright Mary A nn Liebert, Inc.; V iral } \\
\text { Immunol ogy is avail lable online at: http://online.liebertpub.com. }\end{array}$ \\
\hline Type & article \\
\hline File Information & V iral Immunol_27(6)_285-294.pdf \\
\hline
\end{tabular}

Instructions for use 


\title{
The J6JFH1 Strain of Hepatitis C Virus Infects Human B-Cells with Low Replication Efficacy
}

\author{
Masato Nakai,, ${ }^{1,2}$ Tsukasa Seya, Misako Matsumoto, Kunitada Shimotohno, ${ }^{3}$ \\ Naoya Sakamoto, ${ }^{2}$ and Hussein H. Aly ${ }^{1, *}$
}

\begin{abstract}
Hepatitis $\mathrm{C}$ virus (HCV) infection is a serious health problem worldwide that can lead to hepatocellular carcinoma or end-stage liver disease. Current treatment with pegylated interferon, ribavirin, and NS3/4A protease inhibitor would lead to a good prognosis in a large population of patients, but there is still no effective vaccine for HCV. HCV robustly infects hepatocytes in the liver. However, extrahepatic manifestations such as mixed cryoglobulinemia, a systemic immune complex-mediated disorder characterized by B-cell proliferation, which may evolve into overt B-cell non-Hodgkin's lymphoma, have been demonstrated. HCV-RNA is often found to be associated with peripheral blood lymphocytes, suggesting a possible interaction with peripheral blood mononuclear cells (PBMCs), especially B-cells with HCV. B-cell HCV infection was a matter of debate for a long time, and the new advance in HCV in vitro infectious systems suggest that exosome can transmit HCV genome to support "infection." We aimed to clarify the susceptibility of primary B-cells to HCV infection, and to study its functional effect. In this article, we found that the recombinant HCV J6JFH1 strain could infect human B-cells isolated from the peripheral blood of normal volunteers by the detection of both HCV-negative-strand RNA by reverse transcription polymerase chain reaction, and NS5A protein. We also show the blocking of HCV replication by type I interferon after B-cell HCV infection. Although HCV replication in B-lymphocytes showed lower efficiency, in comparison with hepatocyte line (Huh7) cells, our results clearly demonstrate that human Blymphocytes without other non-B-cells can actually be infected with HCV, and that this interaction leads to the induction of B-cells' innate immune response, and change the response of these cells to apoptosis.
\end{abstract}

\section{Introduction}

C HRONIC INFECTION BY HEPATITIS C VIRUS (HCV) is the major cause of liver cirrhosis and hepatocellular carcinoma. About $3.1 \%$ of the global population is infected with HCV (50). Historically, a combination therapy with pegylated interferon (IFN) and ribavirin was used for patients infected with genotype $1 \mathrm{HCV}$. NS3/4A protease inhibitors were recently developed in addition to pegylated IFN and ribavirin, and their combinations have been clinically tried for $\mathrm{HCV}$ treatment since then. Although $>70 \%$ of patients with high viral loads of HCV genotype $1 \mathrm{~b}$ have a sustained viral response by the therapy using simeprevir or telaplevir with pegylated IFN and ribavirin $(17,22)$, the remaining patients fail to eliminate the virus, and drug resistance remains an issue that must be resolved. Recent development of directacting antiviral (DAA) drugs (such as daclatasvir, asuna- previr, and sofosbuvir) are a promising therapeutic option beyond IFN in the treatment of HCV patients $(6,32)$.

$\mathrm{HCV}$ is a single-stranded, positive-sense RNA virus in the Hepacivirus genus of the Flaviviridae family. Although $\mathrm{HCV}$ is known to infect hepatocytes in the liver and induce hepatitis in vivo, in vitro cultured primary hepatocytes barely support the HCV life cycle: only hepatoma Huh7 cells and its subclones can efficiently maintain the HCV life cycle of a very limited number of HCV strains in vitro (53).

Chronic hepatitis patients with HCV sometimes show other extrahepatic complications such as lymphoproliferative diseases (LPD), including cryoglobulinemia and B-cell malignant lymphoma, autoimmune diseases, and dermatitis $(1,12,15,16)$. Epidemiological analysis shows that chronic $\mathrm{HCV}$ patients have higher rates of LPDs than non-HCVinfected populations $(36,48,52)$. Several reports suggested that some lymphotropic HCV strains effectively infected human

\footnotetext{
Departments of ${ }^{1}$ Microbiology and Immunology, and ${ }^{2}$ Gastroenterology, Hokkaido University Graduate School of Medicine, Kita-ku, Japan.

${ }^{3}$ Research Center for Hepatitis and Immunology, National Center for Global Health and Medicine, Ichikawa, Japan.

*Present affiliation: Department of Virology II, National Institute of Infectious Diseases, Toyama, Tokyo, Japan.
} 
lymphocytes $(20,47)$, leading to the above-mentioned abnormalities. Infection of lymphocytes with HCV has been a matter of debate for a long time. More than one decade ago, several reports described the existence of HCV-RNA in peripheral blood mononucleated cells (PBMCs) $(30,40)$. The detection rate of HCV-RNA in PBMCs was increased if patients were infected with human immunodeficiency virus (HIV) together with HCV (44). This phenomenon indicated that immune-suppressive circumstances and/or HIV antigen might enhance the replication activity of HCV in lymphoid cells (44). Moreover, it was reported that continuous release of $\mathrm{HCV}$ by PBMCs was detected in HCV-infected patients, especially in HIV co-infected patients (7). In addition to HCV-HIV co-infected patients, a low level of HCV replication could be detected in peripheral lymphoid cells from $\mathrm{HCV}$ mono-infected patients after antiviral treatment $(34,45)$. Moreover, it was reported that $\mathrm{HCV}$ persisting at low levels long after therapy-induced resolution of chronic hepatitis $\mathrm{C}$ remained infectious (34). This continuous viral presence could present a risk of infection reactivation.

It has been reported that HCV replication was detected in various kinds of lymphoid cells. Many reports describing the existence of $\mathrm{HCV}$ in B-lymphocytes and B-cell lymphoma have been published $(21,25,51)$. Among B-lymphocytes, $\mathrm{CD} 27+$ memory B-lymphocytes were more resistant to apoptosis than CD27 - B-lymphocytes. CD27 + B-lymphocytes were reported as a candidate subset of the $\mathrm{HCV}$ reservoir in chronic hepatitis $\mathrm{C}(\mathrm{CH}-\mathrm{C})$ (38). On the other hand, others claimed that distinguishing RNA association from true $\mathrm{HCV}$ replication was problematic, together with multiple artifacts complicated detection and quantitation of the replicative intermediate minus strand RNA $(29,31)$, and also the failure of retroviral (37) and lentiviral (8) pseudoparticles bearing $\mathrm{HCV}$ envelope glycoproteins (HCVpp) to infect primary Bcells or B-cell lines. This led to continuous debate about $\mathrm{HCV}$ infection into B-lymphocytes, and the riddle remained unsolved.

Using the recent progress in $\mathrm{HCV}$ infection systems, we intended to clarify this debate and analyze HCV infection in human lymphocytes and its functional results. Here, albeit in a lower efficiency compared to HCV infection into Huh7 cells, we report that two different strains of recombinant $\mathrm{HCV}$ viruses could infect primary human lymphocytes not only by the detection of HCV-RNA positive and negative strands proliferation, but also NS5A protein detection, and the detection of the activity of luciferase reporter encoded by the recombinant $\mathrm{HCV}$-genome. Blocking of $\mathrm{HCV}$ entry using anti-CD81 antibody (Ab), and replication by IFN- $\alpha$ or NS3/4A protease inhibitors successfully suppressed $\mathrm{HCV}$ infection. We also found that HCV infection into B-lymphocytes led to the initiation of host response including apoptosis resistance.

\section{Materials and Methods}

\section{Cells and reagents}

Huh7.5.1 cells were kindly provided by Dr. Francis V Chisari (The Scripps Research Institute, La Jolla, CA). Cells were cultured in high-glucose Dulbecco's modified Eagle's medium (DMEM ; Gibco/Invitrogen, Tokyo, Japan) supplemented with $2 \mathrm{mM}$ L-Glutamine, $100 \mathrm{U}$ of penicillin $/ \mathrm{mL}$, $100 \mu \mathrm{g}$ of streptomycin/mL, $1 \times$ MEM non-essential amino acid (Gibco/Invitrogen), and 10\% fetal bovine serum (FBS).
Human peripheral blood mononuclear cells (PBMCs) were obtained from healthy volunteers by density gradient centrifugation using Ficoll Paque plus (GE-Healthcare, Waukesha, WI). CD19+ blood cells (representative of human primary B-cells) and CD19 - cells (non-B-cells) were separated by MACS CD19 Beads (Milteny Biotec, Bergisch Gladbach, Germany). Purity of CD19+ B-cells was $>95 \%$ after two-cycle separation. The cells were cultured in RPMI1640 (Gibco/Invitrogen) supplemented with $100 \mathrm{U}$ of penicillin $/ \mathrm{mL}, 100 \mu \mathrm{g}$ of streptomycin $/ \mathrm{mL}$, and $10 \%$ FBS.

The following reagents were obtained as indicated: antiCD81 Ab (BD Pharmingen, Franklin Lakes, NJ); PE antiCD80 Ab, APC anti-CD86 Ab, and PE-labeled anti-CD19 $\mathrm{Ab}$ (eBioscience, San Diego, CA); recombinant IFN- $\alpha$ (Peprotech, Oak Park, CA); BILN2601 (Behringer, Willich, Germany); and Viaprobe 7AAD (BD Bioscience) and Annexin-V-Fluos (Roche, Mannheim, Germany).

\section{Virus propagation}

pJ6-N2X-JFH1 was kindly provided from Dr. Takaji Wakita (National Institute of Infectious Diseases, Tokyo) (2). pJc1-GLuc2A was gifted from Dr. Brett D. Lindenbach (Yale University, New Haven) (41). In vitro RNA transcription, gene transfection into Huh7.5.1 cells, and preparation of J6JFH1 and Jc1/GLuc2A viruses were performed as previously reported (53). Briefly, the HCV cDNA in plasmids were digested by XBaI and transcribed by T7 Megascript Kit (Invitrogen, Carlsbad, CA). RNA transfection into Huh7.5.1 was performed by electroporation using Gene Pulser II (Bio-Rad, Berkeley, CA) at $260 \mathrm{~V}$ and 950 Cap. Culture supernatant were collected on days $3,5,7$, and 9 of postelectroporation, and concentrated with an Amicon Ultra-15 Centrifugal Filter unit (Millipore, Billerica, MA). The titer of HCVcc was checked by the immunofluoresence method using NS5A antibody when Huh7.5.1 was reinfected with these HCVcc.

\section{Virus infection}

Primary B-cells and non-B-cells were cultured with the J6JFH1 HCV strain at a multiplicity of infection $(\mathrm{MOI})=1-$ 3 for $3 \mathrm{~h}$, and cells were harvested after four extensive washes in culture medium. On days $1-6$, cells were collected, washed with $0.25 \%$ trypsin-EDTA/saline, and incubated with $0.25 \%$ trypsin-EDTA for $5 \mathrm{~min}$ at $37^{\circ} \mathrm{C}$. Then, suspended cells were collected as a source of total RNA. In some experiments, B-cells were infected with the Jc1/ GLuc2A strain at MOI $=5$ for $3 \mathrm{~h}$. Cells were washed five times in $1 \times$ phosphate buffered saline (PBS), and cultured until day 6 for determination of viral replication as GLuc activity with BioLux Gaussia luciferase assay kits (41).

\section{RNA purification, RT-PCR, and quantitative $P C R$}

Total RNA was extracted by using Trizol Reagent (Invitrogen) according to the manufacturer's instructions. Using $100-400 \mathrm{ng}$ of total RNA as a template, we performed RTPCR and real-time RT-PCR as previously described $(3,4)$. Primer sets are shown in Supplementary Table S1 and Table S2 (Supplementary Data are available online at www.lie bertpub.com/vim).

Real-time PCR was used for quantification of positivestrand and negative-strand HCV RNA. Total Trizol-extracted 
RNA was analyzed by RT-PCR with a modification of the previously described strand-specific rTth RT-PCR method $(10,13)$. RT primers for complementary DNA synthesis of positive and negative strand HCV RNA are shown in Supplementary Table S1. Positive-strand and negative-strand HCV PCR amplifications were performed using Power SYBR Green PCR Master Mix (Applied Biosystems, Warrington, UK) with $200 \mathrm{nM}$ of paired primers (Supplementary Table S1). The PCR conditions were $95^{\circ} \mathrm{C}$ for $10 \mathrm{~min}$, followed by 40 cycles at $95^{\circ} \mathrm{C}$ for $15 \mathrm{sec}$ and $60^{\circ} \mathrm{C}$ for $1 \mathrm{~min}$.

\section{Virus production and releasing assay}

Primary human B-cells were infected with J6JFH1 at $\mathrm{MOI}=1$. Six days postinfection, the supernatant was collected ("releasing samples"), cells were repeatedly frozen and thawed, and the supernatant was collected ("assembly samples"). Viral titers of "releasing samples" and "assembly samples" were determined with Huh7.5.1 cells using J6JFH1 virus $(\mathrm{MOI}=0.001$ and 0.01$)$ as control. Total RNA was recovered from the cells on days 2,4 , and 6 , and determined with HCV-RNA to check reinfectivity.

\section{Indirect immunofluorescence}

Indirect immunofluorescence (IF) expression of $\mathrm{HCV}$ proteins was detected in the infected cells using rabbit IgG anti-NS5A antibody (Cl-1) (3). Goat anti-rabbit Alexa 594 (Invitrogen) was used as secondary Ab. Fluorescence detection was performed on the Zeiss LSM 510 Meta confocal microscope (Zeiss, Jena, Germany) (13).

\section{Luciferase assay}

Primary B-cells were infected with Jc1/Gluc2A by using concentrated Medium or Mock Medium (PBS-electropolated Huh7.5.1 medium). Media were collected on days 0 , 2,4 , and 6 postinfection, cleared by centrifugation $(16,000 \mathrm{~g}$ for $5 \mathrm{~min}$ ), and mixed with 0.25 volume of Renilla 5 lysis buffer (Promega, Madison, WI) to kill HCV infectivity. GLuc activity was measured on a Berthold Centro LB 960 luminescent plate reader (Berthold Technologies, Bad Wildbad, Germany) with each $20 \mu \mathrm{L}$ sample injected with $50 \mu \mathrm{L}$ BilLux Gaussia Luciferase Assay reagent (New England Biolabs, Ipswich, MA), integrated over $1 \mathrm{sec}$.

\section{Cell survival assay}

Apoptosis assay: Primary B cells were infected with J6JFH1 virus. Cells were collected $48 \mathrm{~h}$ after infection, stained by 7AAD Cell Viability assay kit and Annexin V, and analyzed by FACS Calibur (BD) (13).

\section{ATP assay}

Primary B-cells were infected with J6JFH1 virus or Mock concentrated medium. Cells were resuspended and cultured at Lumine plate (Berthold Technologies) postinfection. ATP activities were determined $72 \mathrm{~h}$ later using CellTiter-Glo ${ }^{\circledR}$ Luminescent Cell Viability Assay (Promega) according to the manufacturer's protocol.

\section{miRNA detection}

Total RNA was extracted by using Qiazol Reagent (Invitrogen). These RNA was purificated and reverse transcripted to cDNA by using the miScript II RT Kit. Synthesized cDNA was used to determine the expression levels of miR-122 (24). Total miRNA was prepared by using Qiazol and miScript II RT kit (Invitrogen), and miR-122 expression was determined by using miScript SYBR Green PCR Kit and miScript Primer Assay (Invitrogen) according to the manufacturer's protocol. U6 small nuclear RNA was used as an internal control.

\section{Results}

\section{J6JFH1 infects and replicates in primary B-cells}

To address HCV infectivity into primary B-cells, PBMC were isolated from the blood of healthy volunteers and were sorted into CD19+ cells (primary B-lymphocytes) and CD19 - cells (non-B-cells). Their purities were $>95 \%$. These cells were then incubated with the J6JFH1 HCV. Total RNA was collected on days 2, 4, and 6. The Huh7.5.1 strain was used as positive control. Both Huh7.5.1 and primary Bcells, but not non-B-cells, showed an increase in intracellular HCV-RNA titer, albeit primary B-cells showed lower efficiency than Huh7.5.1 (Fig. 1A). We adjusted the HCVRNA values using GAPDH as an internal control (Fig. 1B). To confirm J6JFH1 replication in primary B-cells using IF, we also measured the expression of HCV-NS5A, which is a nonstructural protein produced only by the virus secondary to replication. Although the expression was far lower than Huh7.5.1 cells, we managed to detect the NS5A expression in J6JFH1 infected primary B-cells (Fig. 1C).

We examined what kinds of HCV-entry receptors human primary B-cells expressed in our setting. Human CD81, SRB1, and NPC1L1 were expressed, but not the tight junction proteins claudin 1 and occludin in mRNA levels (Supplementary Fig. S1). We could not detect miR122 in primary B-cells (Supplementary Fig. S2), expression of which makes the cells permissive to HCV (24). Human CD81 is a primary entry receptor for $\mathrm{HCV}$ in hepatocytes (42). Blocking human CD81 by its specific Ab resulted in blockage of HCV infection into primary B-cells, as shown by the suppression of HCV-RNA titer (Fig. 2), suggesting that HCVcc particles enter B-cells also using CD81 receptor. HCV-RNA titer was not suppressed by non-specific Ab (data not shown).

We then examined the effect of the different drugs used to suppress HCV replication (recombinant human IFN, and HCV protease inhibitor, BILN2601). Inhibition of HCV-RNA replication was observed when B-cells were treated with rhIFN- $\alpha$ or BILN2601 (Fig. 2) after infection. BILN2601 showed efficient inhibitory effect on replication of $\mathrm{HCV}$ RNA in Huh7.5.1 cells (Supplementary Fig. S3). As control studies, we confirmed that the production of HCV RNA was reduced in Huh7.5.1 cells by CD81 Ab, IFN- $\alpha$, or BLIN2601 (Supplementary Fig. S4). In both Huh 7.5.1 and B-cells, BLIN2601 most effectively block HCV replication. These data reinforce that $\mathrm{HCV}$ is actually replicating in primary B-cells, and that activation of innate immunity by IFN treatment or blocking the NS3/4A protease function is a critical factor in blocking $\mathrm{HCV}$ replication in primary B-cells. These data suggest that our system can be used for screening the function of different inhibitors on HCV replication in B-cells.

\section{HCV negative-strand RNA detected in human B-cells}

To confirm HCV replication in primary B-cells further, we tested for an increase of negative-strand HCV-RNA after 


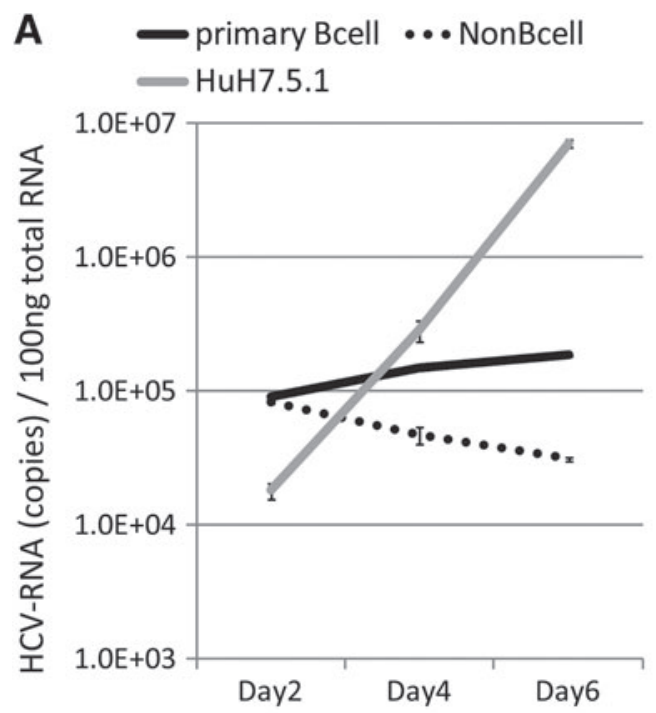

C

Primary Bcell : No infection
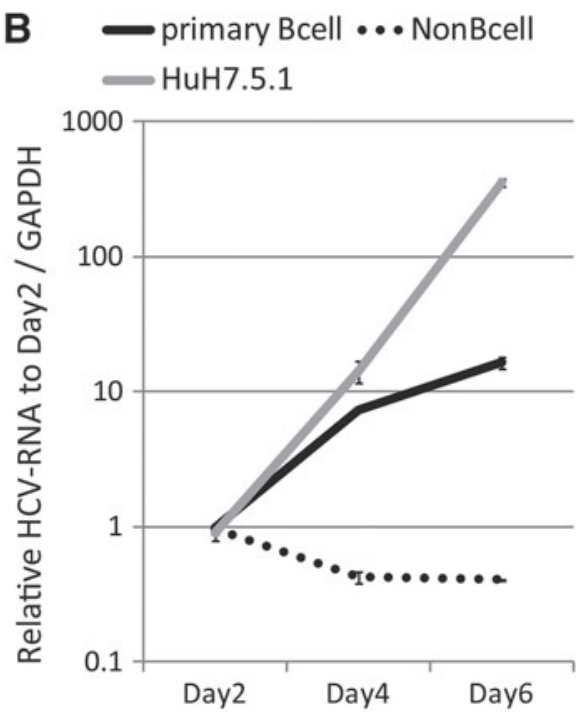

HuH7.5.1 : J6JFH1

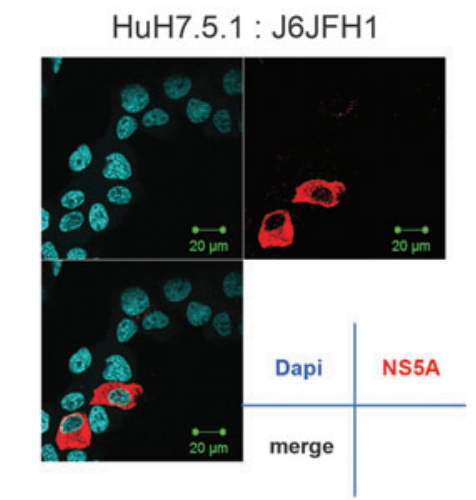

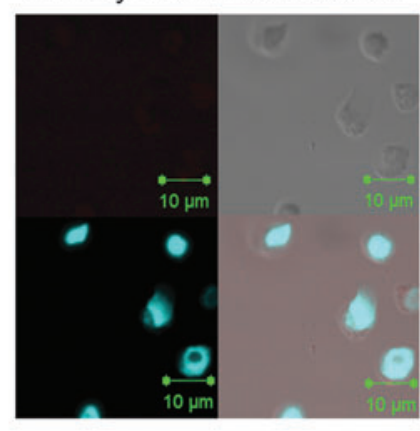
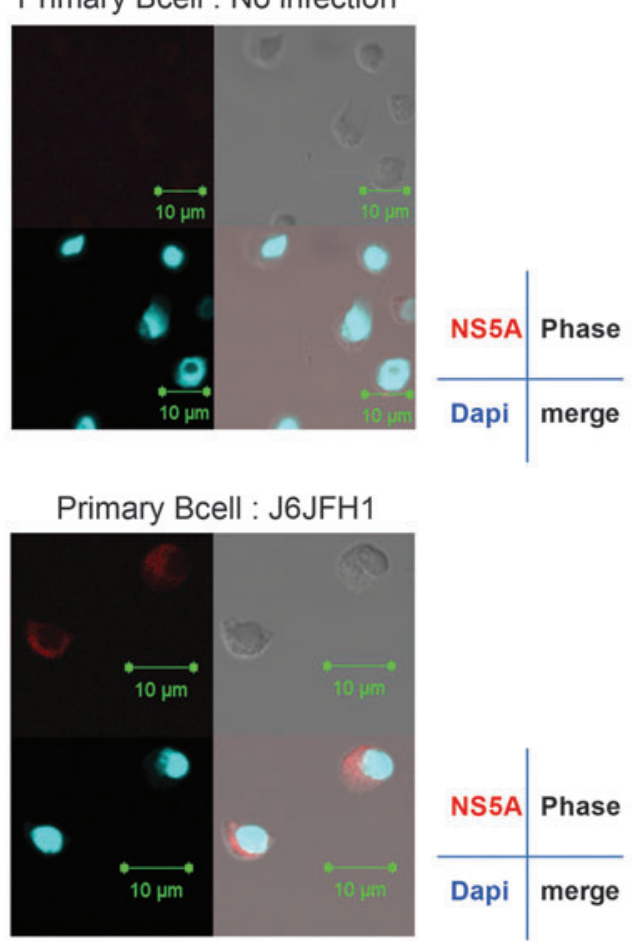

FIG. 1. J6JFH1 infects human peripheral blood B-cells. Human B-cells (CD19+ cells) and non-B-cells (CD19- cells) were separated by MACS as described in Materials and Methods. Primary B-cells, non-B-cells, and Huh7.5.1 cells were infected with $\mathrm{J} 6 \mathrm{JFH} 1$ at $\mathrm{MOI}=1$ for $3 \mathrm{~h}$. After infection, cells were washed twice with culture medium and continued culture. On days 2, 4, and 6, total RNA was collected and HCV-derived RNA was determined by reverse transcription polymerase chain reaction (RT-PCR). GAPDH was used as internal control. (A) HCV-RNA not adjusted by GAPDH. (B) HCV-RNA adjusted by GAPDH. (C) Immunofluorescence analysis of J6JFH1-infected human B-cells and Huh7.5.1 cells. Six days postinfection. Red, NS5A; blue: Dapi; phase: phase-shift microscope.

infection, since the negative-strand RNA is not yielded if HCV particles or RNA just adhere to the cell surface of human primary B-cells without internalization $(9,14,19,35$, 42,43). We measured the synthesis of plus-strand and minus-strand HCV-RNA separately using strand-specific RT primers and rTth polymerase as previously described (4). The titer increase of minus-strand HCV-RNA indicates HCV-RNA replication. As shown in Figure 3, both minus- and plus-strand HCV-RNA increased time dependently in primary B-cells, and both types of RNA concomitantly decreased in non-B-cells (Fig. 3A and B). Plus- and minusstrand RNA were exponentially increased in Huh7.5.1 cells infected with J6JFH1 (Fig. 3C). These results indicated that primary human B-cells supported J6JFH1 infection and replication, although viral replication levels in B-cells were modest compared with those in Huh7.5.1 cells. These results 


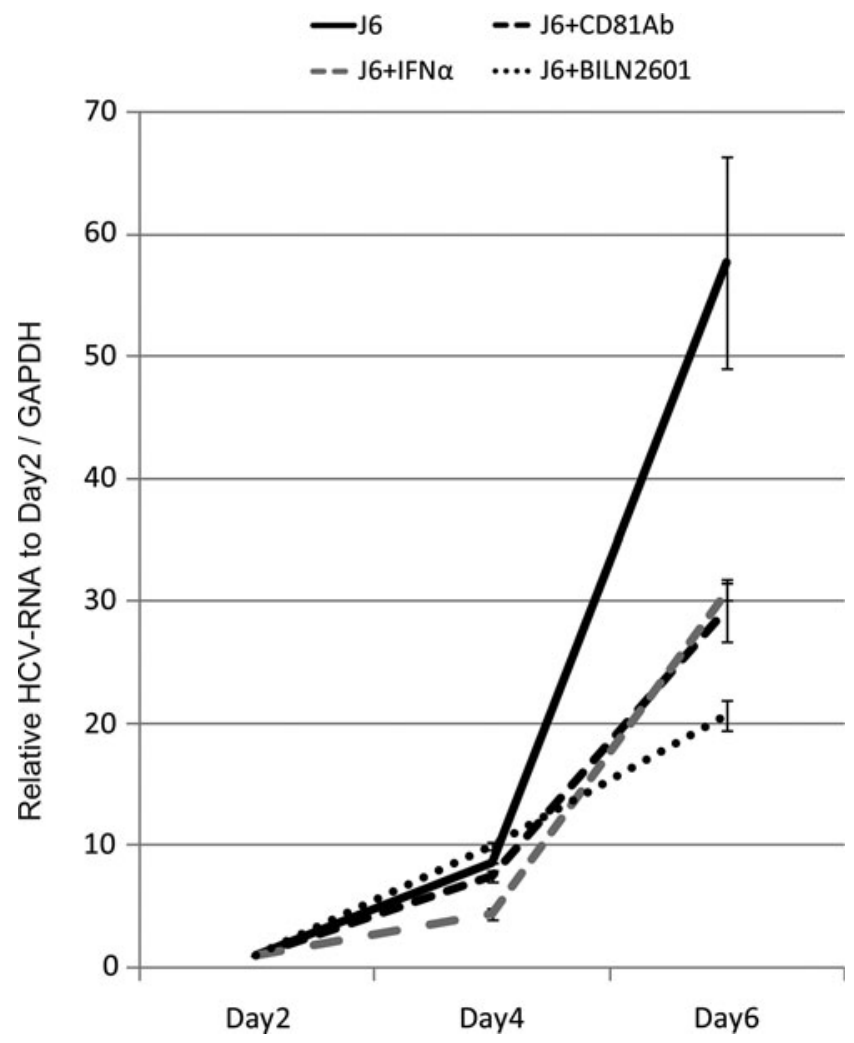

FIG. 2. J6JFH1 B-cell infection is blocked by anti-CD81 $\mathrm{Ab}, \mathrm{IFN}-\alpha$, or an NS3/4A inhibitor. Anti-CD81 neutralizing $\mathrm{Ab}(20 \mu \mathrm{g} / \mathrm{mL})$ was added to the B-cell culture $1 \mathrm{~h}$ before infection. Otherwise, recombinant IFN- $\alpha$ rhIFN- $\alpha$, $200 \mathrm{IU} /$ $\mathrm{mL})$ or BLIN2601 (250 nM, which is IC75; see Supplementary Fig. S3) was added $1 \mathrm{~h}$ after infection. On days 2, 4, and 6, total RNA was extracted, and HCV-RNA was determined by RT-PCR. The values were adjusted by GAPDH.

may reflect the fact that the NS5A protein is difficult to detect in infected B-cells using IF assay.

\section{B-cells can be infected with different HCV strains}

We next used the Jc1/GLuc2A strain to investigate whether different HCV strains infect primary B-cells. Primary Bcells, non-B-cells (data not shown), and Huh7.5.1 cells were infected with the Jc1/GLuc2A strain. After five washes, supernatant was collected (day 0 samples). On days 2, 4, and 6, medium was collected. Luciferase activity was determined for all samples by luminescence (GLuc). GLuc activity and detection of RNA increased exponentially in Huh7.5.1 cells infected with the Jc1/GLuc2A strain (Fig. 4A). GLuc activity on day 4 to day 6 increased more in primary B-cells than in non-B-cells (Fig. 4B). These results suggest that HCV replication is substantial, but low in the HCV line Jc1/GLuc2A.

\section{$B$-cells neither produce nor release detectable level of $\mathrm{HCV}$ infectious particles}

We collected supernatants of J6JFH1-infected primary human B-cells to measure productive infection in B-cells. The supernatant was then added to culture of Huh7.5.1 cells, and we compared infection with control Huh7.5.1 cells, whose cells were infected with a low MOI (0.01 and 0.001) of J6JFH1 collected from media of the infected Huh7.5.1 cells. HCV-RNA titer in the Huh7.5.1 titrating cells was decreased over time after co-culture with B-cell supernatants obtained from either "releasing samples" "assembly samples." In contrast, HCV-RNA titers were slightly increased over time in the Huh7.5.1 titrating cells that had been infected with medium collected from low MOI-J6JFH1-infected Huh7.5.1 cells (Fig. 5). These results indicated that primary human B-cells were infected with J6JFH1 but failed to assemble or produce particles into the supernatant.

\section{Host response to $\mathrm{HCV}$ infection into primary B-cells}

Next, we determined whether B-cell activation was induced in $\mathrm{HCV}$-infected B-cells that survived under $\mathrm{HCV}$ infection. We measured induction of CD80 and CD86 as Bcell activation markers. After 2-3 days of infection, the CD80/86 levels on B-cells treated with J6JFH1 were compared with those treated with medium from mock-infected cells (concentrated Huh7.5.1 medium) by FACS analysis (Fig. 6A). We found that CD80/86 were upregulated in infected cells compared to mock-infected cells.

Since B-cell lymphoma is a known complication of chronic HCV infection $(20,36)$ and acquiring apoptotic resistance is essential for the development of cancer $(21,51,38)$, we measured the ability of B-cells to escape apoptosis after $\mathrm{HCV}$ infection. B-cell apoptosis spontaneously occurs during culture at $37^{\circ} \mathrm{C}$. The percent of apoptosis of primary B-cells was decreased in FACS analysis using 7AAD viaprobe + annexinV (Fig. 6B) and ATP assays postinfection (Fig. 6C). These results suggest that primary B-cells are protected from apoptosis by infection with HCVcc. It has been reported that B-cells were vulnerable to apoptotic cell death at various stages of peripheral differentiation and during signal responses (18). Thus, the results infer that HCV stimulation interferes with B-cell apoptotic signal in human B-cells.

\section{Discussion}

We show evidence suggesting that human peripheral B-cells can be infected with HCV strains. Establishment of J6JFH1 infection was evaluated by minus-strand PCR amplification, production of core and NS5A proteins, and protection from apoptosis. An increase in HCV RNA in Bcells was inhibited by an exogenously added antibody against CD81 that blocked HCV receptor function. Furthermore, blocking $\mathrm{HCV}$ replication in B-cells by type I IFN and NS3/4A protease inhibitor confirmed the presence of $\mathrm{HCV}$ infection/replication in human B-cells. The results were corroborated with another HCV strain, Jc1/GLuc2A. Although we failed to establish an EBV-transformed B-cell line to reproduce $\mathrm{HCV}$ infection of B-cells, peripheral blood B-cells were infected with J6JFH1 in 12 independent experiments.

One of the well-known complications of chronic $\mathrm{HCV}$ infection is LPD, including cryoglobulinemia and B-cell malignant lymphoma, indicating the involvement of B-cells in the course of the disease $(1,12,15,16)$. However, many reports describing the existence of the $\mathrm{HCV}$ genome in B-cells and lymphomas $(21,25,51)$ and HCV replication in B-cells have been controversial due to multiple artifacts complicated in detection and quantitation of the replicative 
A
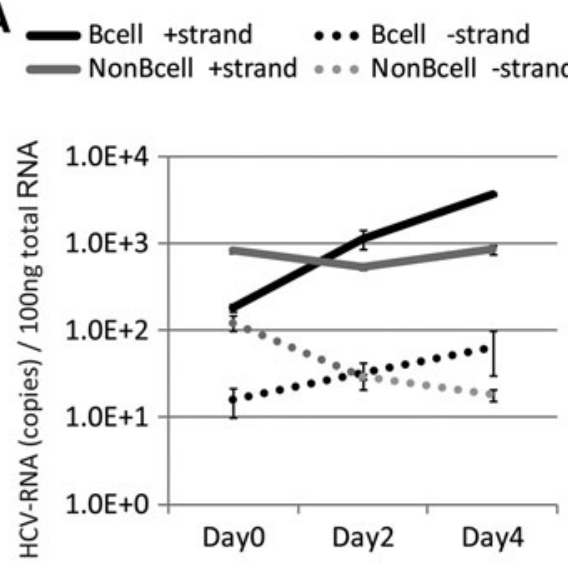

C

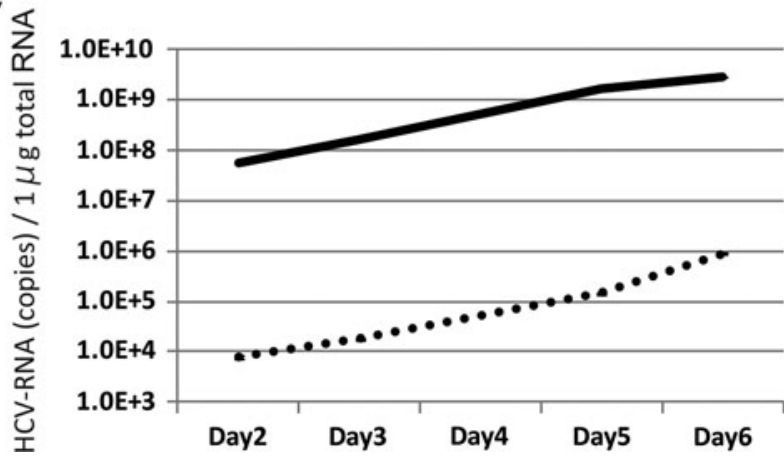

B - Bcell +strand $\quad$... Bcell -strand

NonBcell +strand $\ldots$ NonBcell -strand

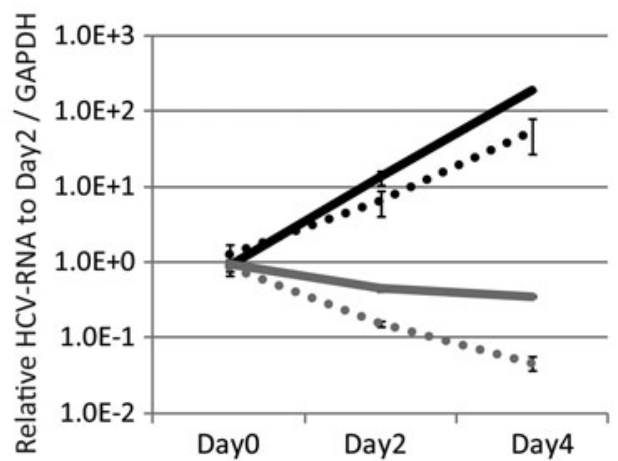

FIG. 3. HCV negative strand RNA is detected in human B-cells. By using rTth methods, HCV strand-specific RNA was determined in J6JFH1-infected human B-cells. (A) Not only plus strand HCV-RNA but also minus strand HCV-RNA were increased in a time-dependent manner in human B-cells. (B) When HCV-RNA was adjusted by GAPDH that was used as an internal control, HCV-RNAs in B-cells were substantially increased compared with those in non-B-cells. (C) Plus and minus strand HCV-RNAs were efficiently amplified in J6JFH1-infected Huh 7.5.1 cells. The level of HCV-RNA exponentially increased in this hepatocyte line.

intermediate minus strand RNA $(29,31)$. This has led to a continuous debate about HCV infection in B-lymphocytes.

$\mathrm{HCV}$ entry into B-cells has also been previously reported to be absent because retroviral (37) and lentiviral (8) pseudoparticles bearing $\mathrm{HCV}$ envelope glycoproteins (HCVpp) did not infect primary B-cells or B-cell lines. In our study, while we succeeded in infecting Huh7.5.1 cells efficiently with retroviral pseudoparticles for expressing both HCV E1/E2 and the control VSV-G, we failed to establish the same infection in B-cells, suggesting that the block of pseudoparticle entry into B-cells is not related to HCV glycoproteins alone.

Total PBMCs reportedly facilitate $\mathrm{HCV}$ attachment but not internalization (42), so HCV infection of B-cells is abrogated in total PBMCs (35). The cause of HCV absorption is unclear, but incomplete sets of HCV receptors in non-B PBMC cells permit attachment of HCV without internalization. B-cells possess CD81, SRBI, LDL-R, and NPC1L1. Because B-cells are not adherent cells, they do not express claudin 1 and occludin, which forms a receptor complex for HCV $(9,14,19,43)$. Claudin 1 and occludin are components of tight junctions and serve as HCV receptors in human hepatocytes. In infection studies using cells expressing these proteins, however, claudin 1 and occludin only upgrade infection efficacy and are dispensable to infection (5), al- though CD81 is essential for establishment of infection (42). Lack of claudin 1 and occludin or miR122 might be a cause of the low HCV infection efficiency observed in human Bcells. Function blocking of CD81 by its specific antibody suppressed HCV infection in primary B-lymphocytes, which imply that HCV entry into primary B-lymphocyte is dependent on the direct interaction phenomenon between HCV virus particles and CD81 receptor and is not mediated by other nonspecific (CD81 independent) pathways such as exosomal transfer of HCV from Huh7 cells to nonhepatic cells, such as dendritic cells (46).

Previous report using in vitro prepared recombinant $\mathrm{HCV}$ JFH1 particles (HCVcc) failed to establish HCV infection in B-lymphocyte cell lines (39). While HCV is known to infect human hepatocytes in vivo leading to chronic viral hepatitis, in the in vitro conditions, only the combination between Huh7 cells and its derived clones supported robust replication and infection with only JFH1 or its derived chimeras (5). Neither hepatocyte cell lines including primary hepatocytes nor other $\mathrm{HCV}$ strains could reproduce $\mathrm{HCV}$ infection efficiently in vitro (5). These data suggest that the clonal selection of HCV quasispecies by hepatoma Huh7 cells is essential for this robust infection in vitro. The situation would be similar to the JFH1 story in B-cell HCV infection. 


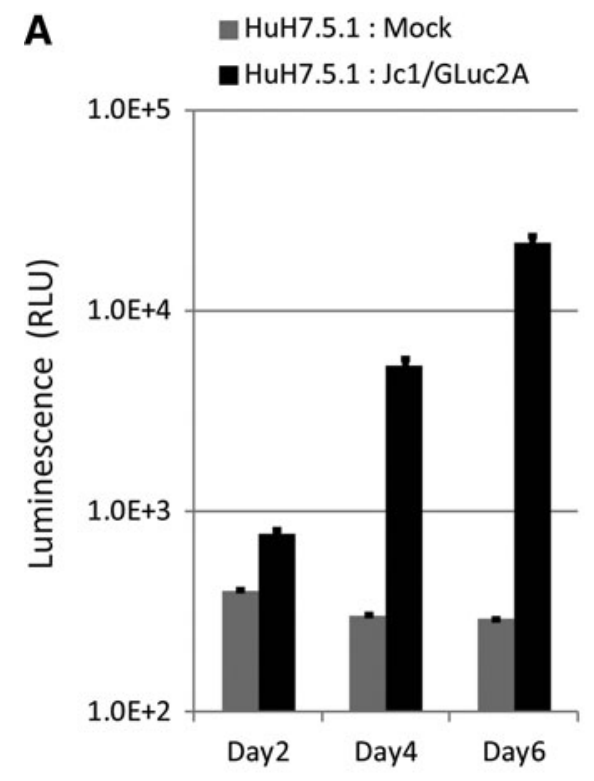

B

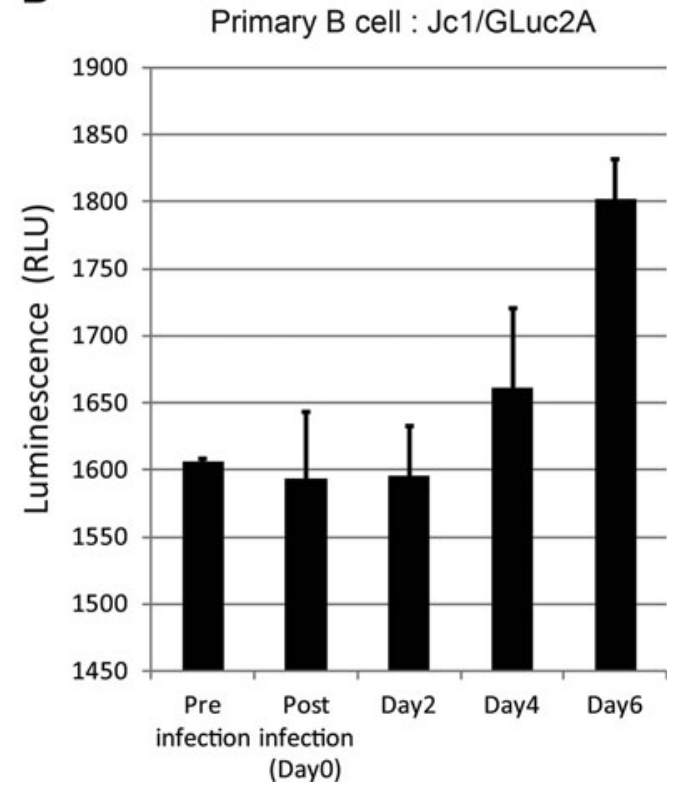

FIG. 4. Jc1/GLuc2A strain infects human B-cells with an increase of Gluc activity. Human B-cells and Huh7.5.1 cells were infected with the JC1/GLuc2A strain that contains secretory luciferase derived from Gaussia $(\mathrm{GLuc})$ at MOI=5. Huh7.5.1 cells were used as control. GLuc activity was increased as time cultured. The GLuc activity was saturated in Huh7.5.1 (A). On the other hand, GLuc activity was increased from day 4 in human B-cells (B).

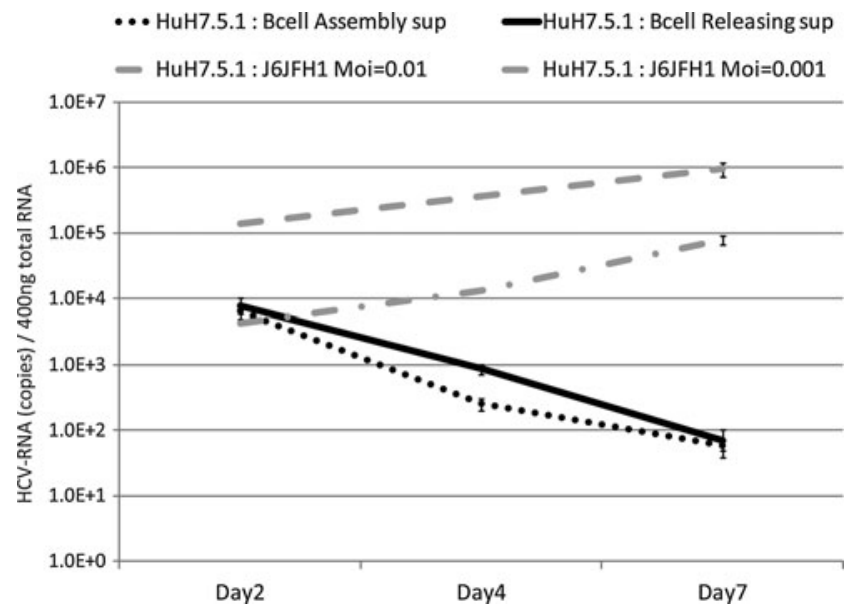

FIG. 5. B-cells infected with J6JFH1 fail to produce virus particles. Human B-cells were infected with J6JFH1 for $3 \mathrm{~h}$, washed twice with phosphate buffered saline (PBS), and cultured. Six days after infection, the supernatant was collected ("releasing samples"). Cells were periodically frozen and thawed five times, and the supernatant was collected ("assembly samples"). For evaluation of the infectious virions, Huh7.5.1 cells were treated with these "releasing samples" or "assembly samples." Similarly, Huh7.5.1 cells were treated with J6JFH1 at low MOI $(\mathrm{MOI}=0.01$ and 0.001) in parallel. After the treatment, cells were washed and cultured. On days 2, 4, and 7, cells were harvested to collect HCV-RNA. Total RNA was extracted from each samples, and HCV-RNA was determined by RT-PCR methods.
B-cell apoptosis spontaneously occurs during culture at $37^{\circ} \mathrm{C}$. We found that $\mathrm{B}$-cell apoptosis was blocked by J6JFH1 infection, as reported previously using Raji cells (11). B-cell apoptosis usually occurs secondary to viral infection, but HCV is particular since apoptotic signaling interferes with infection, leading to protection from cell death. However, B-cell survival was not due to primary infection, because the percent of cells circumventing apoptosis was usually higher than cells infected with HCV. We could not define the pathways that participated in apoptosis regulation by $\mathrm{HCV}$, although a previous report (11) suggested that E2CD81 engagement was related to B-lymphocyte disorders and weak neutralizing antibody response in HCV patients. Since B-cell lymphoma is a known complication of chronic HCV infection (27), the inability of infected cells to undergo apoptosis can be associated with the development of cancer $(28,33,49)$. In this context, B-cell lymphoma often occurs in mice with Cre-initiated HCV transgenes (26). It is notable that anti-apoptotic effect of HCV core gene was reported in genotype 3a in Huh7 cells (23) and, here, genotype 2a in B-cells. In another report (51), HCV strains established from B-cell lymphoma persistently infected with HCV were genotype $2 \mathrm{~b}$. B-cell HCV infection might not be linked to some specific genotypes of HCV.

We believe that our report shows that human primary $\mathrm{B}$-cells can be infected in vitro with $\mathrm{HCV}$, and that this infection is dependent on HCV particles binding with its receptor CD81 and is not nonspecific entry (e.g., exosomal mediated). We also show that this infection could be blocked with antibodies interfering with this binding, or with drugs that suppress HCV replication. Although no virion was generated from B-cells in $\mathrm{HCV}$ infection, it is still likely that B-cells serve as a temporal reservoir of $\mathrm{HCV}$ in the blood circulation. If B-cells permit $\mathrm{HCV}$ infection, RNA sensors 
A

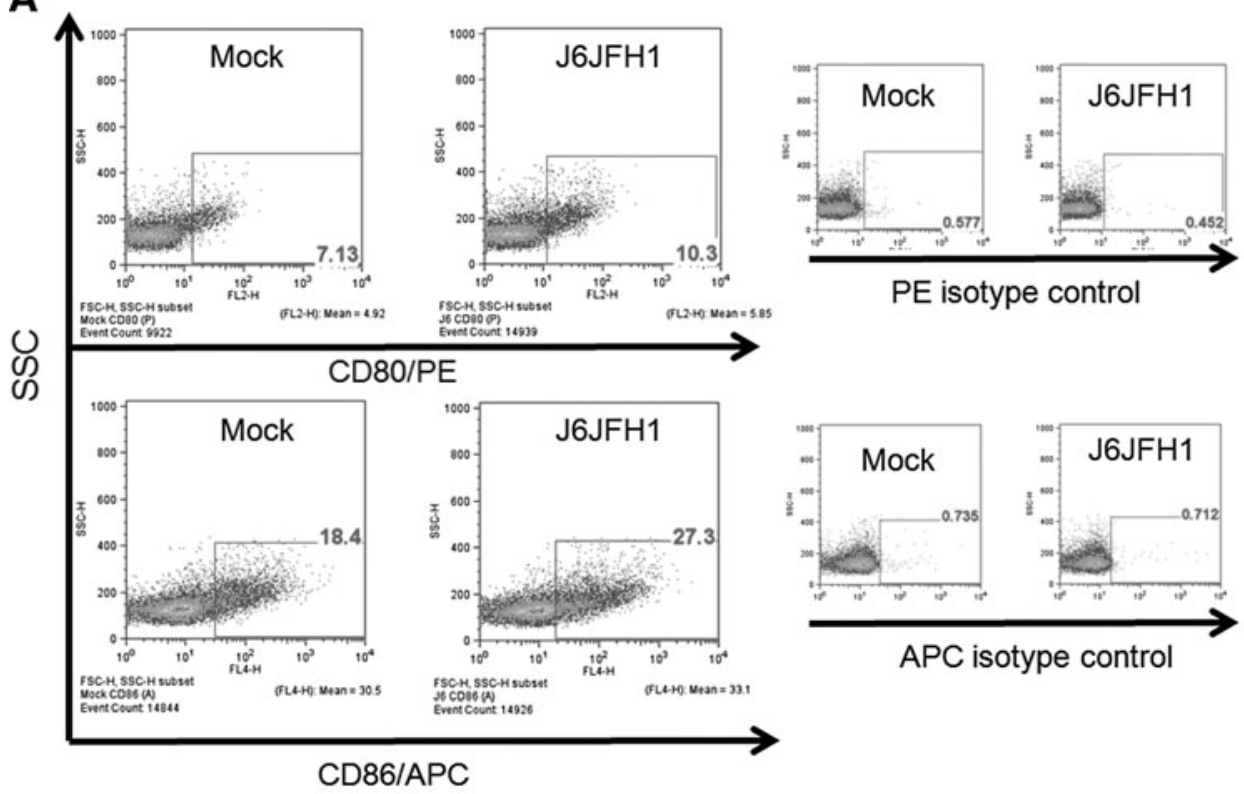

B

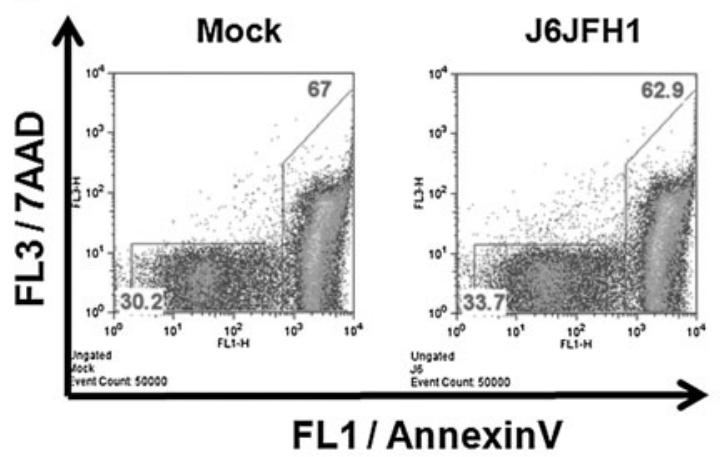

C

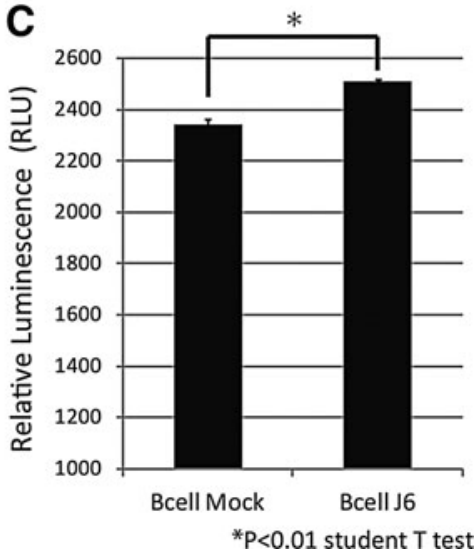

FIG. 6. J6JFH1 infection activates B-cells and protects the cells from apoptosis. Human B-cells were infected with J6JFH1 at $\mathrm{MOI}=1$ for $3 \mathrm{~h}$, washed twice with PBS, and cultured. Two days after inoculation, cells were washed and suspended with FACS buffer. (A) The cells were incubated with PE-conjugated anti-human CD80 antibody, APC-conjugated CD86 antibody, or PE/APC-conjugated mouse IgG1 isotype control for $30 \mathrm{~min}$. Then, the cells were washed and resuspended in FACS buffer. Cells were analyzed by FACS. (B) Annexin V and 7AAD viaprobe were added and cultured at $18^{\circ} \mathrm{C}$ for $10 \mathrm{~min}$. Then, cells were analyzed by FACS. (C) $2 \times 10^{5}$ human B-cells were infected with J6JFH1- or Mock-concentrated medium for 3 h. Cells were then washed, resuspended, and cultured in a 96-well white microwell plate. Two days later, ATP activity was determined with a CellTiter-Glo ${ }^{\circledR}$ Luminescent Cell Viability Assay Kit (Promega). ATP activity was adjusted by day 0 ATP activity.

RIG-I and MDA5 in B-cells might recognize HCV RNA and evoke intracellular signaling, including by transcription factors NF- $\kappa \mathrm{B}$ and IRF-3/7 (5). Activation of the cytokine network is triggered in human B-cells in response to HCV RNA. In fact, host factors liberated by $\mathrm{HCV}$-infecting B-cells have been previously reported in HCV patients $(1,12,15,16,52)$. Although patients' outcomes would be more than we can be predicted from our results, this system would actually benefit the future study on B-cell-virus interaction.

\section{Acknowledgments}

We are grateful to Drs. Frank Chisari (Scripps Research Institute, San Diego, CA) for the Huh7.5.1 cells, Takaji Wakita (National Institute of Infectious Diseases, Tokyo) for supplying the J6JFH1 plasmid, and Brett Lindenbach (Yale University, New Haven, CT) for providing us with the pJc1-GLuc2A HCV strain.

This work was supported in part by Grants-in-Aid from the Ministry of Education, Science, and Culture (Specified Project for "Carcinogenic Spiral") and the Ministry of Health, Labor, and Welfare of Japan, and by the Program of Founding Research Centers for Emerging and Reemerging Infectious Diseases, MEXT. Financial support by the Takeda Science Foundation, the Yasuda Cancer Research Foundation, and the Iskra Foundation are gratefully acknowledged.

\section{Author Disclosure Statement}

No competing financial interests exist. 


\section{References}

1. Agnello V, Chung RT, and Kaplan LM. A role for hepatitis $\mathrm{C}$ virus infection in type II cryoglobulinemia. N Engl J Med 1992;327:1490-1495.

2. Aizaki H, Morikawa K, Fukasawa M, et al. Critical role of virion-associated cholesterol and sphingolipid in hepatitis $\mathrm{C}$ virus infection. J Virol 2008;82:5715-5724.

3. Aly HH, Oshiumi H, Shime $\mathrm{H}$, et al. Development of mouse hepatocyte lines permissive for hepatitis $\mathrm{C}$ virus (HCV). PLoS One 2011;6:e21284.

4. Aly HH, Qi Y, Atsuzawa K, et al. Strain-dependent viral dynamics and virus-cell interactions in a novel in vitro system supporting the life cycle of blood-borne hepatitis $\mathrm{C}$ virus. Hepatology 2009;50:689-696.

5. Aly HH, Shimotohno K, Hijikata M, and Seya T. In vitro models for analysis of the hepatitis $\mathrm{C}$ virus life cycle. Microbiol Immunol 2012;56:1-9.

6. Asselah T, and Marcellin P. Second-wave IFN-based triple therapy for HCV genotype 1 infection: simeprevir, faldaprevir and sofosbuvir. Liver Int 2014;34:60-68.

7. Bare P, Massud I, Parodi C, et al. Continuous release of hepatitis $\mathrm{C}$ virus (HCV) by peripheral blood mononuclear cells and B-lymphoblastoid cell-line cultures derived from HCV-infected patients. J Gen Virol 2005;86:1717-1727.

8. Bartosch B, Dubuisson J, and Cosset FL. Infectious hepatitis C virus pseudo-particles containing functional E1E2 envelope protein complexes. J Exp Med 2003;197; 633-642.

9. Bartosch B, Vitelli A, Granier C, et al. Cell entry of hepatitis $\mathrm{C}$ virus requires a set of co-receptors that include the CD81 tetraspanin and the SR-B1 scavenger receptor. J Biol Chem 2003;278:41624-41630.

10. Castet V, Fournier C, Soulier A, et al. Alpha interferon inhibits hepatitis $\mathrm{C}$ virus replication in primary human hepatocytes infected in vitro. J Virol 2002;76:8189-8199.

11. Chen Z, Zhu Y, Ren Y, et al. Hepatitis C virus protects human B lymphocytes from Fas-mediated apoptosis via E2CD81 engagement. PLoS One 2011;6:e18933.

12. Donada C, Crucitti A, Donadon V, et al. Systemic manifestations and liver disease in patients with chronic hepatitis C and type II or III mixed cryoglobulinaemia. J Viral Hepat 1998;5:179-185.

13. Ebihara T, Shingai M, Matsumoto M, Wakita T, and Seya T. Hepatitis C virus-infected hepatocytes extrinsically modulate dendritic cell maturation to activate $\mathrm{T}$ cells and natural killer cells. Hepatology 2008;48:48-58.

14. Evans MJ, von Hahn T, Tscherne DM, et al. Claudin-1 is a hepatitis $\mathrm{C}$ virus co-receptor required for a late step in entry. Nature 2007;446:801-805.

15. Ferri C, Caracciolo F, Zignego AL, et al. Hepatitis $\mathrm{C}$ virus infection in patients with non-Hodgkin's lymphoma. Br J Haematol 1994;88:392-394.

16. Frangeul L, Musset L, Cresta P, Cacoub P, Huraux JM, and Lunel F. Hepatitis $\mathrm{C}$ virus genotypes and subtypes in patients with hepatitis $\mathrm{C}$, with and without cryoglobulinemia. J Hepatol 1996;25:427-432.

17. Fried MW, Buti M, Dore GJ, et al. Once-daily simeprevir (TMC435) with Pegylated interferon and ribavirin in treatment-naïve genotype 1 hepatitis $\mathrm{C}$ : the randomized PILLAR Study. Hepatology 2013;58:1918-1929.

18. Harwood NE, and Batista FD. New insights into the early molecular events underlying B cell activation. Immunity 2008;28:609-619.
19. Hsu M, Zhang J, Flint M, et al. Hepatitis C virus glycoproteins mediate $\mathrm{pH}$-dependent cell entry of pseudotyped retroviral particles. Proc Natl Acad Sci U S A 2003;100: 7271-7276.

20. Inokuchi M, Ito T, Uchikoshi M, et al. Infection of B cells with hepatitis $\mathrm{C}$ virus for the development of lymphoproliferative disorders in patients with chronic hepatitis C. J Med Virol 2009;627:619-627.

21. Ito M, Masumi A, Mochida K, et al. Peripheral B cells may serve as a reservoir for persistent hepatitis $\mathrm{C}$ virus infection. J Innate Immun 2010;2:607-617.

22. Jacobson IM, McHutchison JG, Dusheiko G, et al. Telaprevir for previously untreated chronic hepatitis $\mathrm{C}$ virus infection. N Engl J Med 2011;364:2405-2416.

23. Jahan S, Khaliq S, Siddiqi MH, et al. Anti-apoptotic effect of HCV core gene of genotype 3a in Huh-7 cell line. Virol J 2011;8:522.

24. Kambara H, Fukuhara T, Shiokawa M, et al. Establishment of a novel permissive cell line for the propagation of hepatitis $\mathrm{C}$ virus by expression of microRNA miR122. J Virol 2012;86:1382-1393.

25. Karavattathayyil SJ, Kalkeri G, Liu HJ, et al. Detection of hepatitis C virus RNA sequences in B-cell non-Hodgkin lymphoma. Am J Clin Pathol 2000;113:391-398.

26. Kasama Y, Sekiguchi S, Saito M, et al. Persistent expression of the full genome of hepatitis $C$ virus in $B$ cells induces spontaneous development of B-cell lymphomas in vivo. Blood 2010;116;4926-4933.

27. Kondo Y, and Shimosegawa T. Direct effects of hepatitis C virus on the lymphoid cells. World J Gastroenterol 2013; 19:7889-7895.

28. Ladu S, Calvisi DF, Conner EA, Farina M, Factor VM, and Thorgeirsson SS. E2F1 inhibits c-Myc-driven apoptosis via PIK3CA/Akt/mTOR and COX-2 in a mouse model of human liver cancer. Gastroenterology 2008;135:1322-1332.

29. Lanford RE, Chavez D, Chisari FV, and Sureau C. Lack of detection of negative-strand hepatitis $\mathrm{C}$ virus RNA in peripheral blood mononuclear cells and other extrahepatic tissues by the highly strand-specific rTth reverse transcriptase PCR. J Virol 1995;69:8079-8083.

30. Laskus T, Radkowski M, Wang LF, Vargas H, and Rakela $\mathrm{J}$. The presence of active hepatitis $\mathrm{C}$ virus replication in lymphoid tissue in patients coinfected with human immunodeficiency virus type 1. J Infect Dis 1998;178:1189-1192.

31. Lerat H, Berby F, Trabaud MA, et al. Specific detection of hepatitis $\mathrm{C}$ virus minus strand RNA in hematopoietic cells. J Clin Invest 1996;97:845-851.

32. Lok AS, Gardiner DF, Hézode C, et al. Randomized trial of daclatasvir and asunaprevir with or without PegIFN/RBV for hepatitis $\mathrm{C}$ virus genotype 1 null responders. J Hepatrol 2014;60:490-499.

33. Lowe SW, and Lin AW. Apoptosis in cancer. Carcinogenesis 2000;21:485-495.

34. MacParland SA, Pham TN, Guy CS, and Michalak TI. Hepatitis $\mathrm{C}$ virus persisting after clinically apparent sustained virological response to antiviral therapy retains infectivity in vitro. Hepatology 2009;49:1431-1441.

35. Marukian S, Jones CT, Andrus L, et al. Cell culture-produced hepatitis $\mathrm{C}$ virus does not infect peripheral blood mononuclear cells. Hepatology 2008;48:1843-1850.

36. Mazzaro C, Franzin F, Tulissi P, et al. Regression of monoclonal B-cell expansion in patients affected by mixed cryoglobulinemia responsive to alpha-interferon therapy. Cancer 1996;77:2604-2613. 
37. McKeating JA, Zhang LQ, Logvinoff C, et al. Diverse hepatitis $\mathrm{C}$ virus glycoproteins mediate viral infection in a CD81-dependent manner. J Virol 2004;78:8496-8505.

38. Mizuochi T, Ito M, Takai K, and Yamaguchi K. Peripheral blood memory B cells are resistant to apoptosis in chronic hepatitis C patients. Virus Res 2011;155:349-351.

39. Murakami K, Kimura T, Shoji I, et al. Virological characterization of the hepatitis C virus JFH-1 strain in lymphocytic cell lines. J Gen Virol 2008;89:1587-1592.

40. Muratori L, Gibellini D, Lenzi M, et al. Quantification of hepatitis C virus-infected peripheral blood mononuclear cells by in situ reverse transcriptase-polymerase chain reaction. Blood 1996;88:2768-2774.

41. Phan T, Beran RKF, Peters C, Lorenz IC, and Lindenbach BD. Hepatitis $C$ virus NS2 protein contributes to virus particle assembly via opposing epistatic interactions with the E1-E2 glycoprotein and NS3-NS4A enzyme complexes. J Virol 2009;83:8379-8395.

42. Pileri P, Uematsu Y, Campagnoli S, et al. Binding of hepatitis C virus to CD81. Science 1998;282:938-941.

43. Ploss A, Evans MJ, Gaysinskaya VA, et al. Human occludin is a hepatitis $\mathrm{C}$ virus entry factor required for infection of mouse cells. Nature 2009;457:882-886.

44. Qu J, Zhang Q, Li Y, et al. The Tat protein of human immunodeficiency virus-1 enhances hepatitis $\mathrm{C}$ virus replication through interferon gamma-inducible protein-10. BMC Immunol 2012;13:15.

45. Radkowski M, Gallegos-Orozco JF, Jablonska J, et al. Persistence of hepatitis $\mathrm{C}$ virus in patients successfully treated for chronic hepatitis C. Hepatology 2005;41:106114.

46. Ramakrishnaiah V, Thumann C, Fofana I, et al. Exosomemediated transmission of hepatitis $\mathrm{C}$ virus between human hepatoma Huh7.5 cells. Proc Natl Acad Sci U S A 2013; 110:13109-13113.
47. Sarhan MA, Pham TNQ, Chen AY, and Michalak TI. Hepatitis $\mathrm{C}$ virus infection of human $\mathrm{T}$ lymphocytes is mediated by CD5. J Virol 2012;86:3723-3735.

48. Schmidt WN, Stapleton JT, LaBrecque DR, et al. Hepatitis $\mathrm{C}$ virus (HCV) infection and cryoglobulinemia: analysis of whole blood and plasma HCV RNA concentrations and correlation with liver histology. Hepatology 2000;31: 737-744.

49. Schulze-Bergkamen H, Krammer P.H. Apoptosis in cancer-implications for therapy. Semin Oncol 2004;31: 90-119.

50. Seto WK, Lai CL, Fung J, et al. Natural history of chronic hepatitis C: genotype 1 versus genotype 6. J Hepatol 2010; 53:444-448.

51. Sung VM, Shimodaira S, Doughty AL, et al. Establishment of B-cell lymphoma cell lines persistently infected with hepatitis $\mathrm{C}$ virus in vivo and in vitro: the apoptotic effects of virus infection. J Virol 2003;77:2134-2146.

52. Turner NC. Hepatitis C and B-cell lymphoma. Ann Oncol 2003;14:1341-1345.

53. Wakita $\mathrm{T}$, Pietschmann $\mathrm{T}$, Kato $\mathrm{T}$, et al. Production of infectious hepatitis $\mathrm{C}$ virus in tissue culture from a cloned viral genome. Nature Med 2005;11:791-796.

Address correspondence to:

Dr. Tsukasa Seya

Department of Microbiology and Immunology Hokkaido University Graduate School of Medicine

Kita 15, Nishi 7

Kita-ku

Sapporo 060-8638

Japan

E-mail: seya-tu@pop.med.hokudai.ac.jp 